Editorial

\title{
Enzyme-Mediated Stereoselective Synthesis
}

\author{
Stefano Serra \\ C.N.R. Istituto di Chimica del Riconoscimento Molecolare, Via Mancinelli 7, 20131 Milano, Italy; \\ stefano.serra@cnr.it or stefano.serra@polimi.it; Tel.: +39-02-2399-3076
}

Received: 6 August 2019; Accepted: 8 August 2019; Published: 11 September 2019

All of us know very well the importance of the catalysis in organic synthesis. Catalyzed reactions are usually preferred when planning a new synthetic approach and the choice of the proper catalyst is of pivotal relevance.

In this context, the use of biocatalysts in organic synthesis has grown steadily during the last fifty years. Presently, chemists have become accustomed to the idea that the use of enzymes is essential in a modern synthetic laboratory. It does not matter if a given transformation is performed using whole cell microorganisms or using an isolated enzyme. In both cases, the specific activity and selectivity of one or more enzymes is exploited. In fact, biocatalysts allow for performing a number of chemical reactions with high regio- and stereoselectivity and a large number of biocatalyzed industrial processes have already been established.

This Special Issue has been planned in order to collect studies focused on the exploitation of enzyme stereoselectivity for the synthesis of relevant chemicals, such as innovative materials, active pharmaceutical ingredients, natural products, flavours and fragrances, and any other kind of bioactive compounds.

Overall, the Special Issue has gathered six articles and one review.

A first paper from Serra and De Simeis [1] describes the stereoselective synthesis of the enantiomeric forms of the alcohol (2,6,6-trimethyltetrahydro-2H-pyran-2-yl)methanol, which are potential chiral building blocks for the preparation of different natural terpenes. Two different catalytic approaches are reported. The first is based on the stereospecific (+)-10-camphorsulfonic acid catalyzed cyclization of 2-methyl-5-(2-methyloxiran-2-yl)pentan-2-ol enantiomers, which are synthesizable from linalool enantiomers, easily available from the chiral pool. The second synthetic approach is based on the lipase-mediated resolution of the target tetrahydropyranyl alcohol, which is available in racemic form starting from the industrial intermediate, dehydrolinalool. In this work, a large-scale resolution procedure that exploits the opposite enantioselectivity of Novozym ${ }^{\circledR} 435$ lipase and lipase AK in the acetylation reaction of (2,6,6-trimethyltetrahydro-2H-pyran-2-yl)methanol is described. The two enantiomeric forms of the latter alcohol were then employed for the first stereoselective synthesis of both enantiomers of the flavor linaloyl oxide.

Concerning resolution based processes, Boratyński et al. [2] reported a novel microbial approach to the production of enantiomerically enriched aroma compounds based on solid-state fermentation using agroindustrial side-stream feedstocks. Twenty-five filamentous fungi were screened for lipase activity and enantioselective hydrolysis of the racemic flavours 1-phenylethyl acetate, trans and cis whisky lactones, $\gamma$-decalactone, $\delta$-decalactone, and cis-3a,4,7,7a-tetrahydro- $1(3 H)$-isobenzofuranone. Solid-state fermentation was conducted with linseed and rapeseed cakes and the results highlight the potential economic value of solid-state fermentation as an alternative to more expensive processes conducted in submerged fermentation.

Two relevant works exploit the potential of biocatalysed reduction reactions. A first contribution from Brenna et al. [3] describes the enantioselective synthesis of $\beta$-nitroalcohols by enzyme-mediated reduction of $\alpha$-nitroketones. In this work, the use of commercial alcohol dehydrogenases (ADHs) 
for the reduction of aromatic and aliphatic nitroketones is investigated. High conversions and enantioselectivities were achieved with two specific ADHs, affording either the $(S)$ or $(R)$-enantiomer of the corresponding nitroalcohols. The further manipulation of the enantioenriched nitroalcohols into protected aminoalcohols is also described. A second contribution from Gotor-Fernández et al. [4] reports the preparation of chiral 1,4-diaryl-1,4-diols through ADH-catalyzed bioreduction of the corresponding bulky 1,4-diaryl-1,4-diketones. Among the different enzymatic preparations used, ADH from Ralstonia sp. (RasADH) overexpressed in E. coli afforded the best results in terms of conversions and diastereo- and enantiomeric excess, allowing the preparation of a set of 1,4-diaryl-1,4-diols bearing different pattern substitutions in the aromatic ring.

Concerning enantioenriched alcohol derivatives, Serra and De Simeis [5] synthesized natural hydroxy fatty acids by means of a biocatalytic hydration reaction of natural fatty acids. The study describes the use of the probiotic bacterium Lactobacillus rhamnosus (ATCC 53103) as whole-cell biocatalyst for the hydration of the most common unsaturated octadecanoic acids, namely oleic acid, linoleic acid, and linolenic acid. The reaction proceeds with high regio- and stereoselectivity. Only 10-hydroxy derivatives were formed and the resulting $(R)$-10-hydroxystearic acid, (S)-(12Z)-10-hydroxy-octadecenoic acid, and (S)-(12Z,15Z)-10-hydroxy-octadecadienoic acid were obtained in very high enantiomeric purity (ee $>95 \%$ ). The biotransformation protocol is stereoselective, scalable, and holds preparative significance, suitable for the production of high-value flavor ingredients.

Yan et al. [6] report a study on the use of modified pullulan polysaccharide for lipase immobilization. Burkholderia cepacia lipase, immobilized on this new material, showed a very good performance, significantly shortened the reaction equilibrium time in transesterification reaction, and exhibited good operational stability. It was found that the enlarged spherical surface of the particle led to high-immobilized efficiency, resulting in the improvement of enantioselectivity in chiral resolution.

Finally, Yun et al. [7] contribute with a review on the recent advances in synthetic applications of $\omega$-transaminases for the production of chiral amines. Recent developments in protein engineering techniques and incorporation of $\omega$-transaminases in multi-enzymatic cascades are there detailed. This work shows that $\omega$-transaminases are efficient catalysts for the synthesis of enantiomerically pure amines.

Overall, these seven contributions give the reader fresh insights on the use of the biocatalysis for the stereoselective synthesis of some relevant chemical compounds (high value products or chiral building blocks) whose preparation, by means of the classical asymmetric processes, presents many drawbacks or cannot be performed.

Funding: This research was funded by [Cariplo Foundation], grant number [2017-1015 SOAVE (Seed and vegetable Oils Active Valorization through Enzymes)] and by [Regione Lombardia], grant number [228775 VIPCAT (Value Added Innovative Protocols for Catalytic Transformations)].

Conflicts of Interest: The author declares no conflict of interest.

\section{References}

1. Serra, S.; De Simeis, D. Two complementary synthetic approaches to the enantiomeric forms of the chiral building block (2,6,6-trimethyltetrahydro-2H-pyran-2-yl) methanol: Application to the stereospecific preparation of the natural flavor linaloyl oxide. Catalysts 2018, 8, 362. [CrossRef]

2. Boratyński, F.; Szczepańska, E.; Grudniewska, A.; Olejniczak, T. Microbial kinetic resolution of aroma compounds using solid-state fermentation. Catalysts 2018, 8, 28. [CrossRef]

3. Tentori, F.; Brenna, E.; Colombo, D.; Crotti, M.; Gatti, F.G.; Ghezzi, M.C.; Pedrocchi-Fantoni, G. Biocatalytic approach to chiral $\beta$-nitroalcohols by enantioselective alcohol dehydrogenase-mediated reduction of $\alpha$-nitroketones. Catalysts 2018, 8, 308. [CrossRef]

4. Mourelle-Insua, Á.; De Gonzalo, G.; Lavandera, I.; Gotor-Fernández, V. Stereoselective enzymatic reduction of 1,4-diaryl-1,4-diones to the corresponding diols employing alcohol dehydrogenases. Catalysts 2018, 8, 150. [CrossRef] 
5. Serra, S.; De Simeis, D. Use of Lactobacillus rhamnosus (ATCC 53103) as whole-cell biocatalyst for the regio-and stereoselective hydration of oleic, linoleic, and linolenic acid. Catalysts 2018, 8, 109. [CrossRef]

6. Xu, L.; Cui, G.; Ke, C.; Fan, Y.; Yan, Y. Immobilized burkholderia cepacia lipase on ph-responsive pullulan derivatives with improved enantioselectivity in chiral resolution. Catalysts 2018, 8, 13. [CrossRef]

7. Patil, M.D.; Grogan, G.; Bommarius, A.; Yun, H. Recent advances in $\omega$-transaminase-mediated biocatalysis for the enantioselective synthesis of chiral amines. Catalysts 2018, 8, 254. [CrossRef]

(C) 2019 by the author. Licensee MDPI, Basel, Switzerland. This article is an open access article distributed under the terms and conditions of the Creative Commons Attribution (CC BY) license (http://creativecommons.org/licenses/by/4.0/). 